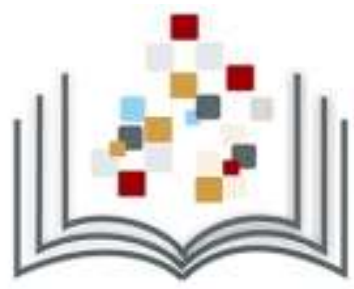

\title{
DA INSPIRAÇÃO AO PROJETO REMAT: UMA CAMINHADA COLABORATIVA E COOPERATIVA EM PROL DO DIÁLOGO CIENTÍFICO
}

Greice da Silva Lorenzzetti Andreis ${ }^{1 *}$, Daiane Scopel Boff ${ }^{1}$, David Matos Milhomens ${ }^{2}$, Henri Luiz Fuchs ${ }^{2}$, Katia Arcaro ${ }^{1}$, Michelsch João da Silva ${ }^{3}$, Rodrigo Sychocki da Silva ${ }^{4}$

1 Instituto Federal de Educação, Ciência e Tecnologia do Rio Grande do Sul - Caxias do Sul (RS) - Brasil

2Instituto Federal de Educação, Ciência e Tecnologia do Rio Grande do Sul - Bento Gonçalves (RS) - Brasil

${ }^{3}$ Instituto Federal de Educação, Ciência e Tecnologia do Rio Grande do Sul - Canoas (RS) - Brasil

4Universidade Federal do Rio Grande do Sul - Departamento de Matemática Pura e Aplicada - Porto Alegre (RS) - Brasil

*Autor correspondente: greice.andreis@caxias.ifrs.edu.br

\section{Como citar:}

ANDREIS, Greice da S. L.. Da inspiração ao projeto remat: uma caminhada colaborativa e cooperativa em prol do diálogo científico. In: WORKSHOP DE EDITORAÇÃO CIENTÍFICA, 9., 2016, São Paulo. Anais... São Paulo: Associação Brasileira de Editores Científicos, 2016. p. 2429

DOI: http://dx.doi.org/10.21452/wec.IXwec.2016.0005

\section{RESUMO}

A Revista Eletrônica da Matemática (REMAT) (e-ISSN: 2447-2689) do Instituto Federal de Educação, Ciência e Tecnologia do Rio Grande do Sul (IFRS), Campus Caxias do Sul, RS, foi criada em 2015, com sua primeira publicação em setembro do mesmo ano. É uma revista de acesso livre, editada e publicada pelo IFRS, Campus Caxias do Sul, em formato eletrônico, no Portal de Periódicos do IFRS ${ }^{1}$ que utiliza o Sistema Eletrônico de Editoração de Revistas (SEER), software desenvolvido para a construção e gestão de publicações periódicas eletrônicas. Apresenta-se em fase de indexação junto ao Google Acadêmico e está cadastrada no Diretório de Políticas Editoriais das Revistas Científicas Brasileiras (Diadorim)2 ${ }^{2}$. As categorias de artigos aceitos para submissão na REMAT são: Ensino de Matemática e Tecnologias Digitais, Matemática na Formação Técnica e/ou Tecnológica e Matemática Pura e Aplicada. Neste artigo, apresentam-se as ideias que inspiraram a criação da REMAT e dados das primeiras três publicações, garantindo o registro histórico do seu primeiro ano de existência.

\section{PERCURSO HISTÓRICO DA REMAT}

A Revista Eletrônica da Matemática (REMAT) foi idealizada no decorrer de 2015 por um grupo de professores do Instituto Federal de Educação, Ciência e Tecnologia do Rio Grande do Sul (IFRS), do Campus Caxias do Sul, atualmente editores científicos e editora-chefe da revista. A inspiração previu a criação de um espaço virtual na web que pudesse armazenar e compartilhar a produção científica do evento Semana Acadêmica da licenciatura em matemática. O evento anual, iniciado em 2011, não tinha até o ano de 2015 um repositório para que as produções provenientes das discussões até o ano

${ }^{1}$ https://periodicos.ifrs.edu.br/

2 http://diadorim.ibict.br/handle/1/1181 
de 2015 um repositório para que as produções provenientes das discussões fossem disponibilizadas. A partir de discussões iniciais entre os professores idealizadores do periódico, a REMAT começou a ser elaborada. O aprimoramento das ideias oportunizou à REMAT o alcance de periódico científico, publicado semestralmente e vinculado ao evento da Semana Acadêmica do Curso de Licenciatura em Matemática.

Sua criação no SEER deu-se em 17 de agosto de 2015. A primeira publicação, realizada em 23 de setembro de 2015, foi totalmente vinculada à Semana Acadêmica do Curso de Licenciatura em Matemática da instituição, que ocorre desde 2011 e constitui-se de um espaço para a divulgação e discussão de práticas de ensino em matemática, minicursos e pesquisas científicas, ligadas à matemática. Esta primeira edição da REMAT foi uma compilação por meio de resumos de algumas das palestras, oficinas, minicursos, relatos de projetos de pesquisa e de extensão, e de trabalhos de conclusão de curso da licenciatura em matemática, realizados nas quatro primeiras edições do evento, que ocorreram nos anos de 2011 a 2014 (ANDREIS; SILVA, 2015). Dos 42 trabalhos apresentados (3 na I, 6 na II, 6 na III e 27 na IV Semana Acadêmica), 14 resumos foram enviados pelos autores e publicados pela REMAT, após avaliações. Esta edição foi organizada em duas seções: Editorial, incluindo a apresentação da edição, e Resumos.

A segunda edição da REMAT teve a submissão de trabalhos de pesquisa oriundos de diversas instituições de ensino. A equipe de avaliadores ad hoc foi ampliada, sendo composta por professores e pesquisadores vinculados à diferentes instituições de ensino nacionais e internacionais. Com isso, a avaliação das produções realizada por esses profissionais ligados tanto ao ensino, quanto à pesquisa, oportunizou a qualificação e o aperfeiçoamento dos textos e, consequentemente, do volume final publicado (ANDREIS et al., 2015). Na ocasião, alguns autores foram convidados a divulgar seus trabalhos na V Semana Acadêmica do Curso de Licenciatura em Matemática, que ocorreu de 3 a 6 de Novembro de 2015. Tal evento teve a finalidade de promover a partilha de experiências entre estudantes e profissionais da área da matemática.

A partir disso, a REMAT, v. 1, n. 2, 2015, com publicação em 5 de janeiro de 2016, foi estruturada em seis seções, sendo elas (ANDREIS et al., 2015): Editorial Apresentação, Corpo Editorial e Avaliadores ad hoc; Palestras - Material fornecido pelos palestrantes que participaram da abertura e do encerramento da $V$ Semana Acadêmica; comunicações científicas - Artigos apresentando resultados parciais ou finais de pesquisas científicas com enfoque no ensino de matemática, matemática aplicada e/ou matemática pura, contribuindo com as pesquisas realizadas nessas subáreas e sua articulação com a prática docente. Parte das comunicações científicas foram apresentadas na V Semana Acadêmica; relatos de experiência - Artigos apresentando uma ação, ou conjunto de ações, que contribuíram com a qualificação das práticas do professor de matemática; oficinas - Artigos contendo uma descrição detalhada das oficinas aplicadas durante a V Semana Acadêmica; resumos - Breves relatos de resultados obtidos em pesquisas científicas ou experiências relacionadas à docência em matemática. Parte dos resumos foram apresentados na V Semana Acadêmica. Nesta edição houve a publicação de 31 trabalhos acadêmicos, sendo distribuídos da seguinte forma: 2 palestras, 7 comunicações científicas, 3 oficinas, 12 relatos de experiência e 7 resumos.

A partir de 2016, as seções da REMAT foram reestruturadas, contando com uma seção especial dedicada à matemática aplicada nos Institutos Federais (IFs), tanto a nível de pesquisa científica quanto a nível de práticas de ensino desenvolvidas na educação básica, nos cursos técnicos, de graduação e de pós-graduação. A primeira edição de 2016, com publicação em 18 de julho de 2016, contou com a submissão de artigos provenientes de diversas instituições de ensino do Brasil (ANDREIS; SILVA, 2016), oportunizando novamente a ampliação do diálogo da comunidade científica. A REMAT, v. 2, n. 1, 2016, foi estruturada com as seguintes seções: Editorial - corpo editorial e 
avaliadores ad hoc; ensino de matemática e tecnologias digitais - Artigos apresentando resultados parciais ou finais originais de pesquisas científicas com enfoque no ensino de matemática, que oportunizam a reflexão sobre a prática docente nas mais diversas modalidades de ensino. Artigos que tratam do uso de tecnologias digitais no ensino de matemática e que apresentam metodologia, resultados e reflexões sobre experimentos realizados em sala de aula; matemática nos IFs - Artigos apresentando resultados parciais ou finais originais de pesquisas científicas desenvolvidas nos IFs com enfoque na matemática. Incluem-se também práticas de ensino de matemática desenvolvidas nos cursos técnicos, de graduação e de pós-graduação, bem como pesquisas que integram a matemática com outros componentes curriculares nos cursos em questão; matemática pura e aplicada - Artigos apresentando resultados parciais ou finais originais de pesquisas científicas com enfoque na matemática pura ou aplicada. Artigos com interfaces na física, química, engenharias, entre outras ciências. Nessa edição foram publicados 14 artigos, distribuídos nas seguintes seções: ensino de matemática e tecnologias digitais (11 artigos), matemática nos IFs (1 artigo) e matemática pura e aplicada (2 artigos).

\section{METODOLOGIA}

Conforme já apresentado neste documento, a revista iniciou em 2015 com a motivação do grupo de docentes do IFRS, Campus Caxias do Sul, de criar um espaço para armazenar as produções das Semanas Acadêmicas do Curso de Licenciatura em Matemática. Para esse processo, o grupo realizou encontros periódicos discutindo o projeto da revista que, para implantação, contou ainda com o suporte de um bibliotecário.

Nos meses subsequentes à criação da REMAT, o grupo de editores, vislumbrando uma revista que tivesse caráter científico e fosse amplamente lida pelos profissionais da área, passou a discutir os processos que norteiam as submissões e avaliações de artigos.

Os artigos são submetidos pelos seus autores cadastrados no sítio eletrônico da REMAT. Os textos submetidos devem seguir as orientações disponibilizadas no site, vinculando-os a uma das seções da revista. A submissão de artigos ocorre em fluxo contínuo. A publicação dos artigos aprovados é realizada no final do primeiro ou segundo semestre de cada ano. Os prazos e as etapas de avaliação são definidos pela Equipe Editorial e publicados no sítio da REMAT. Após a submissão, os artigos são encaminhados para avaliação de pares, às cegas, sem identificação de autoria, sendo esta somente disponível nos metadados acessíveis unicamente aos editores. Os avaliadores ad hoc, após análise criteriosa, emitem seus pareceres indicando uma das seguintes opções: aceitação, revisão requerida, nova submissão ou rejeição do artigo. Os editores estabelecem a comunicação com os autores e encaminham os artigos para revisão, editoração e publicação. A publicação de uma edição da REMAT envolve um expressivo número de docentes pesquisadores que atuam nas tarefas de editoria, avaliação, revisão, formatação e publicação no sítio da revista que conta com a colaboração de autores de diferentes contextos geográficos e educacionais políticas de seção adotadas pela revista:

1. Matemática na formação técnica e/ou tecnológica - artigos apresentando resultados parciais ou finais originais de pesquisas científicas desenvolvidas em cursos técnicos ou tecnológicos com enfoque na matemática. Incluem-se também práticas de ensino de matemática desenvolvidas nos cursos técnicos, de graduação e de pós-graduação, bem como pesquisas que integrem a matemática com outros componentes curriculares nos cursos em questão;

2. Ensino de matemática e tecnologias digitais - artigos apresentando resultados parciais ou finais originais de pesquisas científicas com enfoque no ensino de 
matemática, que oportunizem a reflexão sobre a prática docente nas mais diversas modalidades de ensino. Artigos que tratem do uso de tecnologias digitais no ensino de matemática e que apresentem metodologia, resultados e reflexões sobre experimentos realizados em sala de aula;

3. Matemática pura e aplicada - artigos apresentando resultados parciais ou finais originais de pesquisas científicas com enfoque na matemática pura ou aplicada. Artigos com interfaces na física, química, engenharias, entre outras ciências.

\section{RESULTADOS E DISCUSSÃO}

$\mathrm{Na}$ figura 1 apresenta-se os dados estatísticos da REMAT, fornecidos pelo sistema, sobre submissões, práticas editoriais e usuários para os anos de 2015 e 2016. A edição v. 1, n. 2, 2015 está contabilizada no ano de 2016, pois teve sua publicação em 5 de janeiro de 2016. A taxa de aceite foi maior em 2015 do que em 2016 em função de serem trabalhos convidados para publicação, conforme mencionado na seção "Percurso histórico da REMAT". Observa-se um significativo aumento no número de usuários cadastrados, bem como no número de leitores.

Figura 1: Dados estatísticos de 2015 e 2016 sobre submissões, práticas editoriais e usuários.

\begin{tabular}{|c|c|c|c|}
\hline ANO & $<<2015>>$ & ANO & $\ll<2016$ \\
\hline$\square$ Edições publicadas & 1 & $\square$ Edições publicadas & 2 \\
\hline$\square$ Itens publicados: & 44 & $\square$ Itens publicados: & 21 \\
\hline$\square$ Total de submissões: & 77 & $\square$ Total de submissões: & 76 \\
\hline$\square$ Avaliados pelos pares & 52 & $\square$ Avaliados pelos pares & 47 \\
\hline$\square$ Aceito & $44(85 \%)$ & $\square$ Aceito & $30(64 \%)$ \\
\hline$\square$ Rejeitado & $8(15 \%)$ & $\square$ Rejeitado & $17(36 \%)$ \\
\hline$\square$ Submeter novamente & $(\%)$ & $\square$ Submeter novamente & (\%) \\
\hline$\square$ Tempo de avaliação & 7 & $\square$ Tempo de avaliação & 0 \\
\hline$\square$ Tempo até a publicação & 56 & $\square$ Tempo até a publicação & 73 \\
\hline$\square$ Usuários cadastrados & 174 (169 nova(s)) & $\square$ Usuários cadastrados & $286(112$ nova(s)) \\
\hline$\square$ Leitores cadastrados & 104 (101 nova(s)) & $\square$ Leitores cadastrados & 196 (92 nova(s)) \\
\hline
\end{tabular}

Fonte: REMAT, Estatísticas \& Relatórios. Dados coletados em 13 de outubro de 2016.

De acordo com os relatórios gerados pelo sistema, foram contabilizados 725 acessos aos resumos e 667 acessos aos arquivos PDF/A, conforme mostra o Quadro 1. Os resultados evidenciam um crescimento na qualidade dos trabalhos publicados pela REMAT, uma vez que, os maiores acessos encontram-se na última edição, quando a revista foi reestruturada em três seções, apresentando apenas artigos completos.

Quadro 1: Número de acessos aos resumos e arquivos PDF/A em 2015 e 2016

\begin{tabular}{|c|c|c|}
\hline Edições & $\begin{array}{c}\text { Número de acessos aos } \\
\text { resumos }\end{array}$ & $\begin{array}{c}\text { Número de acessos aos } \\
\text { arquivos PDF/A }\end{array}$ \\
\hline v. 1, n. 1, 2015 & 40 & 21 \\
\hline v. 1, n. 2, 2015 & 120 & 138 \\
\hline v. 2, n. 1, 2016 & 565 & 508 \\
\hline Total & 725 & 667 \\
\hline
\end{tabular}

Fonte: REMAT, Estatísticas \& Relatórios. Dados coletados em 13 de Outubro de 2016. 
Nos gráficos 1 e 2 apresenta-se a distribuição geográfica dos autores e dos avaliadores ad hoc em cada uma das edições da REMAT.

Gráfico 1: Distribuição geográfica dos autores nas três primeiras edições da REMAT.

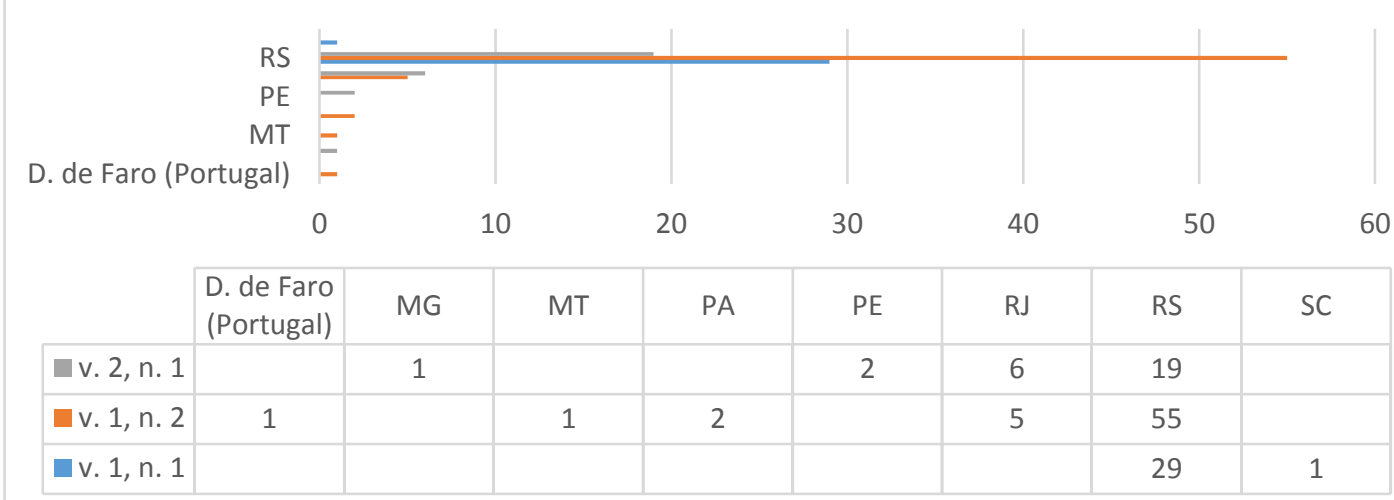

Fonte: REMAT. Dados coletados em 20 de outubro de 2016.

Gráfico 2: Distribuição geográfica dos avaliadores ad hoc nas três primeiras edições.

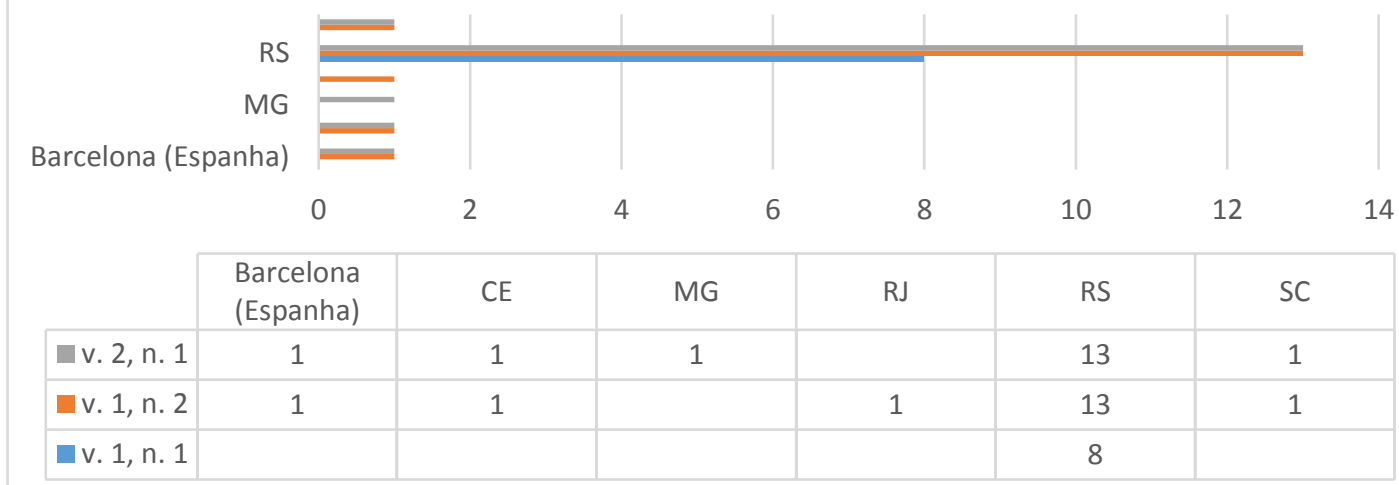

Fonte: REMAT. Dados coletados em 20 de outubro de 2016.

No Quadro 2 apresenta-se os dez artigos mais acessados (arquivo PDF/A) em 2015 e 2016.

Quadro 2: Artigos mais acessados em 2015 e 2016.

\begin{tabular}{|l|c|}
\hline \multicolumn{1}{|c|}{ Artigo } & Acessos \\
\hline $\begin{array}{l}\text { A utilização de resolução de problemas } \\
\text { como estratégia pedagógica no ensino da } \\
\text { matemática no ensino básico }\end{array}$ & 77 \\
\hline $\begin{array}{l}\text { A matemática e o lúdico: ensinando frações } \\
\text { através de jogos }\end{array}$ & 50 \\
\hline $\begin{array}{l}\text { Geometria espacial no ensino fundamental: } \\
\text { construir para aprender }\end{array}$ & 46 \\
\hline $\begin{array}{l}\text { Uso de jogos como estratégia de ensino e } \\
\text { aprendizagem na matemática: estudo de } \\
\text { funções quadráticas no 10 ano do ensino } \\
\text { médio }\end{array}$ & 46 \\
\hline $\begin{array}{l}\text { Explorando funções afins e quadráticas por } \\
\text { meio do software KmPlot com alunos do } \\
\text { ensino médio }\end{array}$ & 36 \\
\hline
\end{tabular}

Fonte: REMAT. Dados coletados em 13 de outubro de 2016. 


\section{CONCLUSÕES}

A construção da REMAT está em constante aperfeiçoamento. Trata-se de uma proposta colaborativa e cooperativa entre os membros de uma comunidade acadêmica, que oportuniza, por meio de um veículo virtual de comunicação, o compartilhamento de ações, experiências e resultados de pesquisas que se relacionem com a matemática. Apesar de estar há pouco tempo inserida no conjunto de periódicos científicos nacionais, a REMAT já alcançou expressividade, manifestada principalmente pelas submissões de manuscritos provenientes de diversas regiões do Brasil. A manutenção e ampliação do projeto permite inferir que a REMAT está trilhando um caminho profícuo para sua consolidação.

A revista tem tomado o direcionamento de atender ao ensino de matemática em contextos educacionais de formação técnica e/ou tecnológica. Pelas primeiras edições percebemos que este é um novo e importante segmento a ser explorado pela comunidade científica, e é o que a REMAT visa atender como eixo principal, sendo este o diferencial da REMAT em relação as revistas já consagradas.

\section{REFERÊNCIAS}

ANDREIS, Greice da Silva Lorenzzetti; SILVA, Rodrigo Sychocki da. Apresentação. REMAT, Caxias do Sul, v. 1, n. 1, 2015.

ANDREIS, Greice da Silva Lorenzzetti; SILVA, Rodrigo Sychocki da; BOFF, Daiane Scopel. Apresentação. REMAT, Caxias do Sul, v. 1, n. 2, 2015.

ANDREIS, Greice da Silva Lorenzzetti; SILVA, Rodrigo Sychocki da. Apresentação. REMAT, Caxias do Sul, v. 2, n. 1, 2016.

PALAVRAS-CHAVE: Histórico. REMAT. Revista Eletrônica. Publicações. 